\title{
A Novel Method for Screening the Effective Compatibility Fractions of Naomaitong for the Treatment of Cerebral Ischemic Stroke Using Support Vector Machine Model
}

Huanzheng Yu

Guangdong Pharmaceutical University

Qiu Liu

Guangdong Pharmaceutical University

Huiting Zheng

BioCR Co.Ltd

Minghua Xian

Guangdong Pharmaceutical University

Shengwang Liang

Guangdong Pharmaceutical University

Shumei Wang ( $\nabla$ gdpuwsm@126.com )

Guangdong Pharmaceutical University

\section{Research}

Keywords: support vector machine (SVM), optimization, Naomaitong (NMT), traditional chinese medicine (TCM)

Posted Date: November 18th, 2020

DOI: https://doi.org/10.21203/rs.3.rs-107304/v1

License: (c) (i) This work is licensed under a Creative Commons Attribution 4.0 International License. Read Full License 


\section{Abstract}

Background: Screening the fractions of traditional Chinese medicine (TCM) medications effective against a disease without affecting their compatibility is a major challenge. Therefore, the goal of the present study was to establish a novel methodology for screening the bioactive fractions of Naomaitong (NMT) utilizing the Support Vector Machine (SVM) model.

Methods: The extract of NMT was separated into 21 fractions using macroporous resin. The different combinations of elution fractions were selected using the Plackett-Burman method and used as an input value for SVM. Infarct size in the rat model of cerebral ischemia-reperfusion injury and the content of selected compounds measured by HPLC were used as output vectors of the SVM training set. Based on the SVM target model, the optimal combination of elution fractions was predicted and verified in the rat model of ischemia-reperfusion injury.

Results: The effective compatibility of NMT was screened by SVM simulation: all elution fractions of rhubarb, $30 \%, 60 \%$, and $95 \%$ ethanol elution fractions of ginseng, and $30 \%$ and $60 \%$ ethanol elution fractions of puerarin and chuanxiong. The optimization of compatibility fractions of NMT was found to have the same protective efficacy in cerebral ischemia-reperfusion injury in rats.

Conclusions: The performed analysis established a novel SVM-based method for screening the compatibility fractions of NMT effective against cerebral ischemic stroke injury. The results provide a new methodology for the optimization of the compatibility of TCM compounds and the discovery of effective components.

\section{Background}

Traditional Chinese medicine (TCM) prescriptions are frequently used in combination with various medicinal herbs to achieve the therapeutic effect of compatibility[1]. The compatibility of TCM prescriptions is essential to generate synergistic effects in clinical treatment[2,3]. However, currently, there are no effective methods for screening fractions of TCM medications effective against a disease, which would not affect their compatibility. In this study, we have used Naomaitong (NMT) as a model to explore a novel method for screening the fractions of NMT effective in the treatment of cerebral ischemic stroke by using the support vector machine model.

Naomaitong (NMT), a Chinese herbal formula, is used clinically in the treatment of cerebral ischemic stroke[4,5]. NMT contains four herbs: Rheum palmatum L. (rhubarb), Panax ginseng C. A. Mey. (ginseng), Pueraria lobata (Willd.) Ohwi. (puerarin), and Ligusticum chuanxiong Hort. (chuanxiong)[6-8]. Although NMT is an effective prescription widely used in China for treating cerebral stroke, its effective fractions are unknown. A crude preparation process, large dosage, and high impurity content in the original NMT preparation, negatively affect its compliance with the requirements for in critical patients, limiting clinical applications of NMT. Therefore, screening and optimizing the effective fraction of NMT is essential to 
develop NMT preparation permitting the use of a lower oral dose and characterized by uniform quality and stable and controllable fractions.

The support vector machine (SVM) prediction model is a method that relies on statistical learning theory to provide optimization $[9,10]$. The advantage of SVM is the small errors obtained using a limited number of training sets still guarantee small errors in the independent test set[11]. An SVM model is to solve optimization problems, which can prevent over-learning. However, how the SVM model can be applied to screening and optimizing the effective fraction of TCM preparations remains unknown. Therefore, the goal of this study was to develop a new SVM-based approach for screening and optimizing the effective fraction of TCM medications using NMT as a study case.

Based on previous research, elution fractions of NMT were prepared using a macroporous resin column[12,13]. To obtain pharmacological data to be used as one of the output vectors of the SVM training set, cerebral ischemia-reperfusion experiments were performed in Sprague-Dawley (SD) rats. The content of representative ingredients in the samples, determined by high-performance liquid chromatography (HPLC), served as another output vector of the SVM training set[9]. Finally, the effectiveness of the optimized formulation of NMT was verified in animal experiments.

\section{Materials And Methods}

\section{Plants}

Rheum palmatum L., Panax ginseng C.A.Mey., Pueraria lobata (Willd.) Ohwi. and Ligusticum chuanxiong Hort. were purchased from Guangzhou Zhixin Pharmaceutical Co., Ltd., and was identified by the expert of Chinese medicine identification of the Guangdong pharmaceutical university, Professor Li Shuyuan.

\section{Chemicals and reagents}

Ethyl acetate, Petroleum ether, trichloromethane, acetic acid, ethyl formate were purchased from Tianjin Zhiyuan Chemical Reagent Co.,Ltd. All the other reagents were of analytic grade. Macroporous resin (AB8) were purchased from Donghong Chemical Co.,Ltd.

\section{Preparation of elution fractions of Naomaitong}

Samples of rhubarb, ginseng, pueraria, and chuanxiong were prepared, with a weight ratio of 9:9:6:6. Chuanxiong medicinal material was immersed in water for $12 \mathrm{~h}$, and steam distilled to extract $4 \mathrm{~h} \sim 8 \mathrm{~h}$ to obtain the volatile oil of chuanxiong[14,15]. The chuanxiong residue and the remaining three fractions were extracted twice by mixing $60 \%$ ethanol for 1 hour. The extracts were combined, filtered, and concentrated to $0.12 \mathrm{~g} / \mathrm{ml}$. Subsequently, the extracts were purified using an AB-8 macroporous resin. The eluents were water, $30 \%$ ethanol, $60 \%$ ethanol, and $95 \%$ ethanol. A total of 17 elution fractions were collected.

\section{Animal grouping}


Male (SD) rats weighing 250-280 g were purchased from Guangdong Medical Laboratory Animal Center. The animals were maintained in specific pathogen-free grade chambers at $22^{\circ} \mathrm{C}$ with a relative humidity of $50 \pm 5 \%$. According to the prescription principle of the NMT compound, the Plackett-Bueman Design method was used to design the table of each group of NMT[16-18], and the method was numerically coded for 17 elution fractions. The factors obtained for each elution fraction are listed in Table 1 and the experimental table (Table 2). According to the following Table 2 for compatibility, in which level 0 indicates "not used" and level 1 indicates "used", 19 experimental groups were designed. Thus, the table contains 21 experimental groups, with one group serving as a model group ("0" for all fractions), and one group treated with all NMT fractions ("1" in all cases).

Table 1 NMT factor level table of each compatibility group $₫ \mathrm{~g} / 100 \mathrm{~g} \rrbracket$

\begin{tabular}{|lllll|}
\hline Elution parts & \multicolumn{3}{l}{ Amount of dry paste added } & \\
\cline { 2 - 5 } & Rhubarb 9g & Ginseng 9g & Chuanxiong 6g & Pueraria 6g \\
\hline Water & $0.301(1)$ & $2.274(5)$ & $1.892(9)$ & $0.966(14)$ \\
\hline $30 \%$ Ethanol & $1.92(2)$ & $0.294(6)$ & $0.09(10)$ & $1.014(15)$ \\
\hline $60 \%$ Ethanol & $0.256(3)$ & $0.25(7)$ & $0.065(11)$ & $0.138(16)$ \\
\hline $95 \%$ Ethanol & $0.085(4)$ & $0.073(8)$ & $0.0012(12)$ & $0.022(17)$ \\
\hline Volatile oil『 $\llbracket \square$ & $/$ & $/$ & $0.0026(13)$ & $/$ \\
\hline
\end{tabular}

Dose unit: $\mathrm{g}(0.3085 \mathrm{~g} / 100 \mathrm{~g}$ according to the raw dose). The unit of volatile oil of ligusticum chuanxiong is $\mathrm{mL} / 100 \mathrm{~g}$. The numbers in brackets represents the coding sequence number of elution fraction.

Table 2 Each compatibility group codes 


\begin{tabular}{|c|c|}
\hline Compatibility group & Coding \\
\hline A & 11010100001101100 \\
\hline B & 10100001101100111 \\
\hline C & 00111101010000110 \\
\hline D & 10011110101000011 \\
\hline$E$ & 01111010100001101 \\
\hline $\mathrm{F}$ & 11001111010100001 \\
\hline G & 01010000110110011 \\
\hline $\mathrm{H}$ & 00110110011110101 \\
\hline I & 11110101000011011 \\
\hline $\mathrm{J}$ & 10000110110011110 \\
\hline K & 01000011011001111 \\
\hline L & 10110011110101000 \\
\hline M & 11101010000110110 \\
\hline$N$ & 01101100111101010 \\
\hline 0 & 00011011001111010 \\
\hline$P$ & 01100111101010000 \\
\hline Q & 10101000011011001 \\
\hline $\mathrm{R}$ & 11011001111010100 \\
\hline 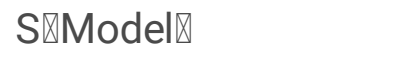 & 00000000000000000 \\
\hline $\mathrm{T}$ & 00001101100111101 \\
\hline Naomaitong & 111111111111111111 \\
\hline
\end{tabular}

The coding order is according to the number of the elution fractions in Table 1. Level 0 means not used, level 1 means used.

The rats were randomly divided into 22 groups, 10 rats each. The groups included a sham operation group, model group, nimodipine $(1.234 \mathrm{mg} / \mathrm{kg})$ group, group treated with all NMT fractions (corresponding to the crude herb dosage of $3.085 \mathrm{~g} / \mathrm{kg}$ ), and 19 groups treated with combinations of NMT (equal to NMT dosage $3.085 \mathrm{~g} / \mathrm{kg}$ ). The drugs were administered for 5 consecutive days before the operation, with the last dose given 2 hours before the surgery[19]. 


\section{The rat model of cerebral ischemia-reperfusion injury}

A modified method of Longa[20,21] was used to establish the Middle cerebral artery occlusion (MCAO) cerebral ischemia-reperfusion rat model. For this purpose, animals were anesthetized by intraperitoneal injection of $7 \%$ chloral hydrate $(300 \mathrm{mg} / \mathrm{kg})$ and placed in the supine position on the operating table. The right common carotid artery (CCA) and external carotid artery (ECA) were separated, making sure that the separation was complete. A small incision was made in the common carotid artery, and a nylon suture was inserted into the CCA and advanced along the internal carotid artery (ICA) for approximately 18-20 $\mathrm{mm}$ until a slight resistance was present at the bifurcation of the CCA. After $2 \mathrm{~h}$ of ischemia, the suture was removed, and the reperfusion was continued for $24 \mathrm{~h}$. For the sham operation, the suture was not inserted.

\section{Infarct area evaluation[22,23]}

After $24 \mathrm{~h}$ of perfusion, rats were anesthetized with $7 \%$ chloral hydrate. The brain was isolated and cut into 2-mm-thick slices. The slice were immersed in a $0.5 \%$ tetrazolium chloride (TTC) solution and incubated at $37^{\circ} \mathrm{C}$ for $15 \mathrm{~min}$. Subsequently, the stained slices were photographed, and the infarct area was measured using Image $\mathrm{J}$ software.

\section{Evaluation of neurological deficits[24,25]}

The neurological deficit test was conducted at $24 \mathrm{~h}$ after the onset of MCAO. The neurological score was evaluated as previously described, and utilized the following scale: grade 0 , no observable deficit; grade 1 , forelimb flexion; grade 2, circling to the left; grade 3, circling to the left in situ; and grade 4, inability to walk.

\section{Content determination of samples}

In accordance with the Chinese Pharmacopoeia (2015 Edition) recommendations for the quantitative evaluation of rhubarb, ginseng, pueraria root, and chuanxiong, HPLC was performed to determine the content of active ingredients in each elution fraction, which included physcion, aloe-emodin, parietic acid, emodin, chrysophanic acid, ginseng Rg1, ginseng Rb1, ginseng Re, puerarin, ferulaic acid and volatile oil.

\section{Data preprocessing}

The different combinations of 21 elution fractions were selected by the Plackett-Burman method as an input value X (Table 3). They were composed of random combinations of all elution fractions of NMT for a total of 17 factors at 2 levels, generating 512 combinations, which constituted the prediction set (Appendix Table 1).

Table $3 \mathrm{X}$-matrix composed of different elution fractions 


\begin{tabular}{|llllllllllllllllll|}
\hline Group & \multicolumn{1}{c|}{ Different elution fractions } & & & & & & & & & & & & & & & & \\
A & 1 & 1 & 0 & 1 & 0 & 1 & 0 & 0 & 0 & 0 & 1 & 1 & 0 & 1 & 1 & 0 & 0 \\
\hline B & 1 & 0 & 1 & 0 & 0 & 0 & 0 & 1 & 1 & 0 & 1 & 1 & 0 & 0 & 1 & 1 & 1 \\
\hline C & 0 & 0 & 1 & 1 & 1 & 1 & 0 & 1 & 0 & 1 & 0 & 0 & 0 & 0 & 1 & 1 & 0 \\
\hline D & 1 & 0 & 0 & 1 & 1 & 1 & 1 & 0 & 1 & 0 & 1 & 0 & 0 & 0 & 0 & 1 & 1 \\
\hline E & 0 & 1 & 1 & 1 & 1 & 0 & 1 & 0 & 1 & 0 & 0 & 0 & 0 & 1 & 1 & 0 & 1 \\
\hline F & 1 & 1 & 0 & 0 & 1 & 1 & 1 & 1 & 0 & 1 & 0 & 1 & 0 & 0 & 0 & 0 & 1 \\
\hline G & 0 & 1 & 0 & 1 & 0 & 0 & 0 & 0 & 1 & 1 & 0 & 1 & 1 & 0 & 0 & 1 & 1 \\
\hline H & 0 & 0 & 1 & 1 & 0 & 1 & 1 & 0 & 0 & 1 & 1 & 1 & 1 & 0 & 1 & 0 & 1 \\
\hline I & 1 & 1 & 1 & 1 & 0 & 1 & 0 & 1 & 0 & 0 & 0 & 0 & 1 & 1 & 0 & 1 & 1 \\
\hline J & 1 & 0 & 0 & 0 & 0 & 1 & 1 & 0 & 1 & 1 & 0 & 0 & 1 & 1 & 1 & 1 & 0 \\
\hline K & 0 & 1 & 0 & 0 & 0 & 0 & 1 & 1 & 0 & 1 & 1 & 0 & 0 & 1 & 1 & 1 & 1 \\
\hline L & 1 & 0 & 1 & 1 & 0 & 0 & 1 & 1 & 1 & 1 & 0 & 1 & 0 & 1 & 0 & 0 & 0 \\
\hline M & 1 & 1 & 1 & 0 & 1 & 0 & 1 & 0 & 0 & 0 & 0 & 1 & 1 & 0 & 1 & 1 & 0 \\
\hline N & 0 & 1 & 1 & 0 & 1 & 1 & 0 & 0 & 1 & 1 & 1 & 1 & 0 & 1 & 0 & 1 & 0 \\
\hline O & 0 & 0 & 0 & 1 & 1 & 0 & 1 & 1 & 0 & 0 & 1 & 1 & 1 & 1 & 0 & 1 & 0 \\
\hline P & 0 & 1 & 1 & 0 & 0 & 1 & 1 & 1 & 1 & 0 & 1 & 0 & 1 & 0 & 0 & 0 & 0 \\
\hline Q & 1 & 0 & 1 & 0 & 1 & 0 & 0 & 0 & 0 & 1 & 1 & 0 & 1 & 1 & 0 & 0 & 1 \\
\hline R & 1 & 1 & 0 & 1 & 1 & 0 & 0 & 1 & 1 & 1 & 1 & 0 & 1 & 0 & 1 & 0 & 0 \\
\hline S & 0 & 0 & 0 & 0 & 0 & 0 & 0 & 0 & 0 & 0 & 0 & 0 & 0 & 0 & 0 & 0 & 0 \\
\hline T & 0 & 0 & 0 & 0 & 1 & 1 & 0 & 1 & 1 & 0 & 0 & 1 & 1 & 1 & 1 & 0 & 1 \\
\hline Z & 1 & 1 & 1 & 1 & 1 & 1 & 1 & 1 & 1 & 1 & 1 & 1 & 1 & 1 & 1 & 1 & 1 \\
\hline
\end{tabular}

From the perspectives of pharmacodynamic indicators and known active ingredients, infarct size in rats subjected to cerebral ischemia-reperfusion and the content of active ingredients were used as output vectors of the SVM training set. To express the difference in the dimension and range of the pharmacodynamic indicators and content of representative ingredients, the data must be normalized. Data normalization eliminates the effect of different measurement units and dimensions on variables, and was performed using the following formula: 


$$
\mathrm{x}(i)=\frac{x_{0}(i)-\operatorname{Min}\left(x_{0}\right)}{\operatorname{Max}\left(x_{0}\right)-\operatorname{Min}\left(x_{0}\right)}
$$

Where $\mathrm{X}$ represents the infarct size of cerebral ischemia-reperfusion rats or the content of representative ingredients. And $Y$ represents the data after normalization, which the vector after normalization was in [0, 1]. Normalization was achieved by the Matlab software using the programming language, $\mathrm{Y}=\operatorname{mapminmax}(\mathrm{X}, 0,1)$.

The content of each elution fraction was normalized and then weighted utilizing the following formula:

$$
\mathrm{n}_{t}=n_{a} \times 0.3+\mathrm{n}_{\mathrm{b}} \times 0.3+n_{c} \times 0.2+\mathrm{n}_{d} \times 0.1+\mathrm{n}_{\mathrm{s}} \times 0.1 \quad \text { (Equations 2) }
$$

Where $n_{t}$ is the result of normalization of the content of the active ingredient in the sample to be administered; $n_{a}$, total anthraquinones; $n_{b}$, total saponins; $n_{c}$, puerarin; $n_{d}$, ferulic acid; $n_{e}$, volatile oil.

The non-infarct area was used as the drug efficacy index and contributed to the calculation. $Y$ was calculated using the following formula:

$$
Y=\mathrm{n}_{t} \times 0.5+\mathrm{m} \times 0.5
$$

(Equations 3)

In which $m$ represents the result after normalization of non-infarct size, and $n_{t}$ represents the result of normalization of the content of the active ingredient.

\section{Establishment of the SVM algorithm model}

The SVM algorithm was calculated using the PLS-SVM toolkits of the Matlab version 13.0. The RBF kernel function was selected, in which the kernel parameter $(\mathrm{Y})$ and the penalty parameter (C) had to be optimized and determined[26,27]. Parameter optimization was performed in the range from $2^{-10}$ to $2^{10}$ using a grid search. To verify the reliability and prediction ability of the mathematical model, the leaveone-out cross-validation method was used, so each sample was used once as a test sample, and n-1 times as a training set sample. Fig. 1 roughly demonstrates the process of SVM screening optimizing combination.

\section{Statistical analysis}

Statistical analysis was performed using Prism software version 6.0 (Graphpad Software, San Diego, California, USA). The data are expressed as the mean \pm S.D. from at least three independent experiments. Statistical significance was determined by one-way analysis of variance (ANOVA). Differences with $p<$ 0.05 were considered statistically significant. 


\section{Results}

\section{Pharmacological Activity of Various Fractions of Naomaitong}

Cerebral infarct size is the most important indicator in the model of cerebral ischemia-reperfusion, and the size of the white area reflects the extent of death of brain nerve cells. In comparison with the sham operation group, the model group had evident infarct (Fig. 2C). Infarct size in the sham operation group, nimodipine group, groups treated with various fractions of NMT was significantly different from the model group $(\mathrm{p}<0.05)$ (Fig.2A). The treatment with nimodipine and with combined NMT fractions had protective effects against ischemia-reperfusion injury. Except for the 0 and $T$ groups, all groups treated with NMT fractions had a significantly smaller size of the cerebral infarct $(p<0.05)$, indicating that different fractions and different compatibility affected the outcome of the ischemic stroke.

\section{Determination of the content of effective components in various fractions of Naomaitong}

Content results measured in the liquid phase (Fig. 3) that the water elution fractions of each herb do not contain the ingredients to be tested. Aloe-emodin and rhein were detected in $30 \%$ and $95 \%$ ethanol elution fractions. Chrysophanol and emodin methyl ether were present mostly in the $60 \%$ ethanol elution fraction. Chrysophanol was mainly concentrated in the $95 \%$ ethanol elution fraction. Ginsenosides $\mathrm{Rg}_{1}, \mathrm{Re}$, and $\mathrm{Rb}_{1}$ were most enriched in the $60 \%$ ethanol elution fraction. Puerarin was concentrated predominantly in the $30 \%$ ethanol elution fraction, and ferulic acid in the $60 \%$ ethanol eluent.

\section{Support vector machine algorithm for the screening of effective compatibility fractions of Naomaitong}

The results of Y-normalization, calculated according to the Y-normalization formula, are listed in Fig. 4.

By assigning Table 3 to $X$ and the normalized results in Fig. 4 to $Y, X$ and $Y$ formed the training set. Using the leave-one-out cross-validation method, the SVM function of the PLS toolkit was applied. The $\mathrm{R}_{1}{ }^{2}$ of the training set of the model was equal to 0.9891 , and the $R_{2}{ }^{2}$ of the prediction set was equal to 0.6182 .

The 512 possible combinations were used as the input vector, $\mathrm{X}_{\text {-predicted, }}$ of the prediction set to obtain the predicted value, $Y_{\text {-predicted }}$ (Appendix Table 1).

\section{Optimized compatibility fractions}

The results listed in Appendix Table 1 (predicted values of 512 combinations of 17 factors) were processed. Each factor was divided into two levels according to the value of the code, level 1 or level 0 , and sorted to obtain two levels of each part. The weight coefficient $\left(Y^{\prime}\right)$ of each fraction at the two levels was obtained, respectively (Fig. 5).

The color indicates the weight coefficient of eluting components at two levels, and tends to be blue, indicating that the eluting component plays a more important role in compatibility. A, water elution site; $B$, $30 \%$ ethanol elution site; C, $60 \%$ ethanol elution site; D, $95 \%$ ethanol elution site; E, volatile oil. 
If the average value of level 1 is greater than the average value of level 0 , the effective part contributes to compatibility; otherwise, the part is invalid. Therefore, the optimized compatibility fractions are comprised of all fractions of rhubarb, $30 \%, 60 \%$, and $95 \%$ ethanol elution fractions of ginseng and chuanxiong, and $30 \%$ and $60 \%$ ethanol elution fractions of pueraria.

\section{Experimental verification of the optimization}

Rats were divided into the sham operation group, model group, nimodipine group, NMT group, and optimization groups with high $(0.617 \mathrm{~g} / 100 \mathrm{~g})$, medium $(0.3085 \mathrm{~g} / 100 \mathrm{~g})$, and low $(0.1543 \mathrm{~g} / 100 \mathrm{~g})$ doses. The experimental protocol was the same as used above.

As shown in Fig. 6, in comparison with the model group, infarct size was significantly reduced in the NMT group, the nimodipine group and the groups treated with low and medium doses $(p<0.05)$ (Fig. 6B). These findings indicate that optimal compatibility fractions were appropriate and effective. However, the infarct size in the optimization groups treated with the high dose was close to the infarct size of the model group, in which diarrhea of adverse reaction occurred. Therefore, based on the determination of the content of individual components (Fig. 3 and Table 4), the results of the SVM optimization are as follows: rhubarb free anthraquinone $221 \mathrm{mg} / \mathrm{kg}$, ginsenoside $688 \mathrm{mg} / \mathrm{kg}$, and ferulic acid $1.06 \mathrm{mg} / \mathrm{kg}, 643 \mathrm{mg} / \mathrm{kg}$ (medium optimized group囚the dosage is calculated according to the crude herb of NMT $3.085 \mathrm{~g} / \mathrm{kg}$ )

Table 4 NMT factor level table of each compatibility group $\mathbb{g} / 100 \mathrm{~g} \rrbracket$

\begin{tabular}{|c|c|c|c|c|}
\hline \multirow[t]{2}{*}{ Elution parts } & \multicolumn{4}{|c|}{ Amount of dry extract powder of different herbs } \\
\hline & Rhubarb 9g & Ginseng $9 g$ & Chuanxiong $6 \mathrm{~g}$ & Pueraria $6 \mathrm{~g}$ \\
\hline Water & 0.301 & 2.274 & 1.892 & 0.966 \\
\hline $30 \%$ Ethanol & 1.92 & 0.294 & 0.09 & 1.014 \\
\hline $60 \%$ Ethanol & 0.256 & 0.25 & 0.065 & 0.138 \\
\hline $95 \%$ Ethanol & 0.085 & 0.073 & 0.0012 & 0.022 \\
\hline Volatile oil $\nabla \mu[$ & / & / & 0.0026 & / \\
\hline Total dry paste & 2.562 & 2.891 & 2.048 & 2.14 \\
\hline
\end{tabular}

Dose unit: g. The unit of volatile oil of chuanxiong is $\mathrm{mL} / 100 \mathrm{~g}$

\section{Discussion}

Screening the effective fractions of traditional Chinese medicine (TCM) formulations is an important part of modernization of TCM. However, to date, there are no effective methods for screening the effective fractions of TCM prescriptions. In the present study, the SVM model was used to identify the fraction of NMT, a representative TCM medication, effective against cerebral ischemic stroke. The result 
demonstrated that the SVM model combining a pharmacological index and the index of the content of effective ingredients could successfully detect effective components of the fractions of TCM formulations.

Chinese herbal compound formula is an important part of TCM and generally comprises two or more types of Chinese herbal medications. Due to their extremely high complexity[28], modern medical therapies continue to face numerous obstacles. SVM screening can be used as a new method to solve this problem. SVM optimizes the effective fraction by analyzing and predicting active components in the Chinese herbal compound formula. The composition of NMT is complex, and its quality is difficult to control. The effective fractions of NMT are not only based on the material basis of the active ingredient, but also reflects its medicinal effect. Therefore冈in the present analysis, we had considered both the pharmacological effect and the content of the active ingredients at the same time. Compared with the previous single-index screening methods, the active ingredients can be screened more comprehensively. In addition, the effective fractions screening follow the theory of traditional Chinese medicine, so as to achieve the purpose of optimizing without changing the pharmacological effect of the original prescription. So NMT fractions were eluted with different concentrations of ethanol separately from different herbs in the guidance of monarch, minister, assistant and guide theory[29,30]. Subsequently, it leads to many experiments to screen the effective fractions. Therefore, the SVM model was combined with Plackett-Burman method to greatly reduce the number of trials, thereby improving the efficiency of screening. Experiments have demonstrated that the main effective ingredients of plants were enriched in the purification process of macroporous resin. According to the published research, the free anthraquinone of rhubarb, both have an effect of treating stroke[31], which is consistent with the experimental results reported here. Ginsenosides and ferulic acid are many reports on the treatment of cerebral bleeding with ginsenoside[32,33], ferulic acid, and chuanxiong volatile oil[34]. The polysaccharide components of the four herbs were mainly eluted in the water, and their effects on stroke rarely reported. The validation experiment documented showed that infarct size was significantly reduced in the low- and medium-dose of the cerebral ischemia-reperfusion group, the nimodipine group, and the NMT group were significantly improved $(p<0.05)$, indicating that the optimized group was assigned reasonable and effective.

Moreover, the creaming rate of polysaccharide of the ginseng, puerarin, and chuanxiong parts constituted more than $50 \%$, and after removing these parts, the creaming rate and the dosage can be markedly reduced. Considering the dry paste conversion data (Table 6), it can be estimated that removing the eluted fractions of ginseng, puerarin, and chuanxiong can reduce the creaming rate by $53 \%$. Elimination of these components would decrease the amount of medication and improve compliance. Together, this study used NMT as an example to screen the effective fractions by the SVM model and demonstrated the model is feasible and adequate. There are still lots of TCM formula that need to be screened for effective fractions, which have many impurities and complicated compatibility. In this way, the SVM model is also suitable for the screening of active ingredients of other TCM, so that provide a new pattern for the optimization of TCM. 


\section{Conclusion}

The effective compatibility of NMT was screened by SVM simulation, which included all elution fractions of rhubarb, $30 \%, 60 \%$, and $95 \%$ ethanol elution fractions of ginseng, $30 \%$ and $60 \%$ ethanol elution fractions of puerarin, and $30 \%$ and $60 \%$ ethanol elution fractions of chuanxiong. In this study, we used the SVM model established by pharmacological index and content of active ingredients index to screen out the effective compatibility of NMT, and the results were verified by animal efficacy. Based on the novel pattern, the active ingredients of effective compatibility fractions are significantly enriched. Verification experiments demonstrated that the effective fractions have the same pharmacological effect on the protection against ischemia-reperfusion injury of the brain in rats as the original prescription.

Moreover, in the case of equivalent efficacy, the dosage can be reduced. The findings indicate that the use of the SVM model to screen the effective compatibility of NMT is effective and feasible, providing a rapid and convenient new technology for the development of novel compound drugs.

\section{List Of Abbreviations}

SVM support vector machine

NMT Naomaitong

TCM traditional chinese medicine

\section{Declarations}

\section{Funding Declarations}

Ethics approval and consent to participate

Not applicable.

\section{Consent for publication}

This manuscript is approved by all authors for publication.

\section{Availability of data and materials}

The datasets generated for this study are available on request to the corresponding author.

\section{Competing interests}

The authors declare that they have no competing interests.

\section{Funding}


This research is supported by Projects of the National Natural Science Foundation of China (grant numbers 81773884, 81473431,81274060) and the National Science and Technology Major Project (grant numbers 2017ZX09301077).

\section{Authors' contributions}

Huanzheng Yu contributed equally to this manuscript. SL and SW conceived and designed the idea; YH and $\mathrm{HZ}$ performed the experiments; QL and XM analyzed the data; MX and $\mathrm{YH}$ wrote the paper. All authors read and approved the final manuscript.

\section{Corresponding authors}

Correspondence to Shengwang Liang or Shumei Wang.

\section{Acknowledgements}

Technology Funds were obtained from the Key Unit of Chinese Medicine Digitalization Quality Evaluation of State Administration of Traditional Chinese Medicine.

\section{References}

1. Zhou X, Seto SW, Chang D, Kiat H, Razmovski-Naumovski V, Chan K, et al. Synergistic Effects of Chinese Herbal Medicine: A Comprehensive Review of Methodology and Current Research. Front Pharmacol [Internet]. 2016; Available from: http://dx.doi.org/10.3389/fphar.2016.00201

2. Zhang J, Zhu Y, Fan X, Zhang B. Efficacy-oriented compatibility for component-based Chinese medicine. Acta Pharmacol Sin [Internet]. 2015; Available from: http://dx.doi.org/10.1038/aps.2015.8

3. Luan X, Zhang L-J, Li X-Q, Rahman K, Zhang H, Chen H-Z, et al. Compound-based Chinese medicine formula: From discovery to compatibility mechanism. J Ethnopharmacol [Internet]. 2020; Available from: http://dx.doi.org/10.1016/j.jep.2020.112687

4. Tan LF, Chen C, Fan XL, Dong Y, Ge YW, Wang SM. Study on pharmacodynamic material basis of Naomaitong to protect neuronal cells based on PK-PD model. China Journal of Chinese Materia Medica. 2019; 44: 2588-93.

5. Wenzhi W, Bin J, Haixin S, Xiaojuan R, Dongling S, Linhong W, et al. Prevalence, Incidence, and Mortality of Stroke in China: Results from a Nationwide Population-Based Survey of 480687 Adults. Pubmed. 2017;135.

6. Rong YY, Feng SX, Liu DY, Wu CW, Liang SW, Wang SM. Identification of chemical constituents in Naomaitong Decoction by HPLCMS/MS. Chinese Traditional Patent Medicine. 2016; 38: 1544-50.

7. Kening L, Minghua X, Chi C, Shengwang L, Lei C, Shumei W. Proteomic Assessment of iTRAQ-Based NaoMaiTong in the Treatment of Ischemic Stroke in Rats. Pubmed. 2019;2019.

8. Deng GZ, Lin JX, Wu CW, Chen C, Wang SM. Molecular docking in Naomaitong Formula preparations multi-target effect on ischemic stroke. Chinese Traditional Patent Medicine. 2016; 38: 1673-78. 
9. Qiao LS, Zhang XB, Jiang LD, Zhang YL, Li GY. Identification of potential ACAT-2 selective inhibitors using pharmacophore, SVM and SVR from Chinese herbs. Mol Divers. 2016;20:933-44.

10. Tang J, Ning J, Liu X, Wu B, Hu R. A Novel Amino Acid Sequence-based Computational Approach to Predicting Cell-penetrating Peptides. Curr Comput Aided Drug Des. 2019;15:206-11.

11. Li SX, Chen C, Wang SM, Deng XH, Liang SW. Simulation Study of Antithrombotic Active Fraction of Naodesheng by Support Vector Machine. Journal of Chinese Medicinal Materials. 2011; 34: 107880.

12. Guo HD, Zhang QF, Chen JG, Shangguang XC, Guo YX. Large scale purification of puerarin from Puerariae Lobatae Radix through resins adsorption and acid hydrolysis. J Chromatogr B Anal Technol Biomed Life Sci. 2015;980:8-15.

13. Ren J, Zheng Y, Lin Z, Han X, Liao W. Macroporous resin purification and characterization of flavonoids from Platycladus orientalis (L.) Franco and their effects on macrophage inflammatory response. Food Funct. 2017;8:86-95.

14. Zhang H, Han T, Yu CH, Jiang YP, Peng C, Ran X, et al. Analysis of the chemical composition, acute toxicity and skin sensitivity of essential oil from rhizomes of Ligusticum chuanxiong. $J$ Ethnopharmacol. 2012;144:791-6.

15. Wei XC, Yang ST, Zhang Y, Pan M, Pan XH, Cheng JM. GC-MS analysis of two assistant methods on extracting volatile oil from Chuanxiong Rhizoma. Chinese Traditional Patent Medicine. 2019; 41: $129-34$.

16. Elazazy MS, El-Hamshary M, Sakr M, Al-Easa HS. Plackett-Burman and Box-Behnken designs as chemometric tools for micro-determination of I-Ornithine. Spectrochim Acta A Mol Biomol Spectrosc. 2018;193:397-406.

17. Nelson BJ, Montgomery DC, Elias RJ, Maass E. A comparison of several design augmentation strategies. John Wiley Sons, Ltd. 2000;16.

18. Sahu AK, Jain V. Screening of process variables using Plackett-Burman design in the fabrication of gedunin-loaded liposomes. Artif Cells Nanomed Biotechnol. 2017;45:1011-22.

19. Wu CW, Guo JW, Chen C, Liang SW, Wang SM. Optimization of component compatibility of naomaitong for anti-cerebral ischemia treatment based on artificial neural network. China Academic Journal Electronic Publishing House. 2016; 51: 454-58.

20. Nan W, Yanmin Z, Lei W, Yuanji W, Yanjun C, Langchong H, et al. Puerarin protected the brain from cerebral ischemia injury via astrocyte apoptosis inhibition. Pubmed. 2014;79.

21. Lu L, Juan C, Yong M, Jianbin L, Dahuan Z, Fei W, et al. Transplantation of Human Umbilical Cord Blood Mononuclear Cells Attenuated Ischemic Injury in MCAO Rats via Inhibition of NF-KB and NLRP3 Inflammasome. Pubmed. 2018;369.

22. Jun Y, Hui Y, Sumei L, Min Z. Berberine Ameliorates MCAO Induced Cerebral Ischemia/Reperfusion Injury via Activation of the BDNF-TrkB-PI3K/Akt Signaling Pathway. Pubmed. 2018;43.

23. Lv B, Feng L, Jianbang H, Jie F, Limin X, Chengmei S, et al. Hif-1a Overexpression Improves Transplanted Bone Mesenchymal Stem Cells Survival in Rat MCAO Stroke Model. Front Mol 
Neurosci. 10.

24. Li M, Wen Y, Zhang R, Xie F, Zhang G, Qin X. Adenoviral vector-induced silencing of RGMa attenuates blood-brain barrier dysfunction in a rat model of MCAO/reperfusion. Elsevier Inc. 2018;142.

25. Litao L, Lipeng D, Zhen X, Weiliang H, Jingru Z, Henan P, et al. Integrated analysis of the proteome and transcriptome in a MCAO mouse model revealed the molecular landscape during stroke progression. Pubmed. 2020;24.

26. Zhang L, Li G, Zhou X, Wu M, Jin M, Zhou Z, et al. Establishment and evaluation of early prognosis models of acute intracerebral hemorrhage based on support vector machine. J Nanjing Med Univ. 2016;

27. Zhao C, Zhang H, Zhang X, Zhang R, Luan F, Liu M, et al. Prediction of milk/plasma drug concentration (M/P) ratio using support vector machine (SVM) method. Pharm Res. 2006;23:41-8.

28. Wang S, Hu Y, Tan W, Wu X, Chen R, Cao J, et al. Compatibility art of traditional Chinese medicine: from the perspective of herb pairs. J Ethnopharmacol. 2012;143:412-23.

29. Lyu C-Y, Lyu S-W, Li G-Y, Kuang H-X. Research progress on pharmacological effects of separation and combination methods based on traditional Chinese medicine property and flavor. Zhongguo Zhong Yao Za Zhi [Internet]. 2018; Available from: http://dx.doi.org/10.19540/j.cnki.cjcmm.20180327.007.

30. Qu J, Zhang T, Liu J, Su Y, Wang H. Considerations for the Quality Control of Newly Registered Traditional Chinese Medicine in China: A Review. J AOAC Int [Internet]. 2019; Available from: http://dx.doi.org/10.5740/jaoacint.18-0301

31. Li Q, Guo Y, Yu X, Liu W, Zhou L. Protective mechanism of rhubarb anthraquinone glycosides in rats with cerebral ischaemia-reperfusion injury: interactions between medicine and intestinal flora. Chin Med. 2020;15:60.

32. Zheng $T$, Jiang $H$, Jin R, Zhao Y, Bai Y, Xu H, et al. Ginsenoside Rg1 attenuates protein aggregation and inflammatory response following cerebral ischemia and reperfusion injury. Eur $\mathrm{J}$ Pharmacol. 2019;853:65-73.

33. CL X, JH L, WW W, GQ Z, LXW. Neuroprotective effect of ginsenoside-Rg1 on cerebral ischemia/reperfusion injury in rats by downregulating protease-activated receptor-1 expression. Life Sci. 2015;121:145-51.

34. Luo L, Kang J, He Q, Qi Y, Chen X, Wang S, et al. A NMR-Based Metabonomics Approach to Determine Protective Effect of a Combination of Multiple Components Derived from Naodesheng on Ischemic Stroke Rats. Molecules [Internet]. 2019;24. Available from: http://www.ncbi.nlm.nih.gov/pubmed/31086027.

\section{Figures}




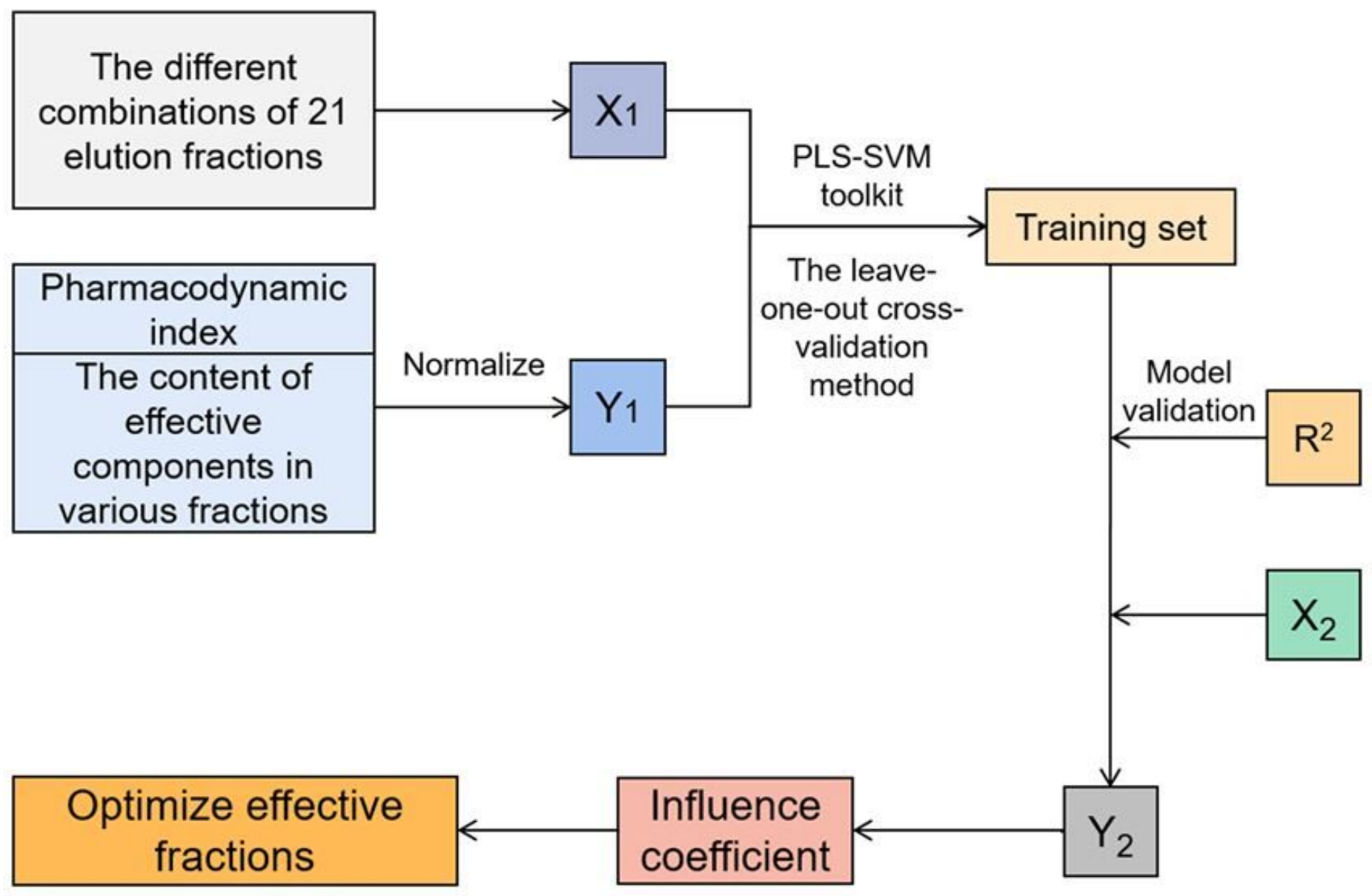

Figure 1

The process of SVM screening optimizing combination. 


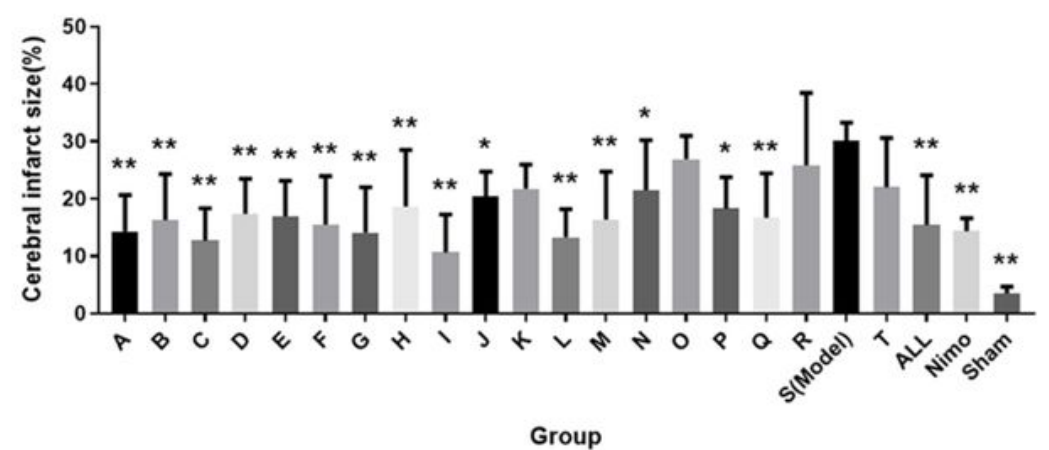

(A)

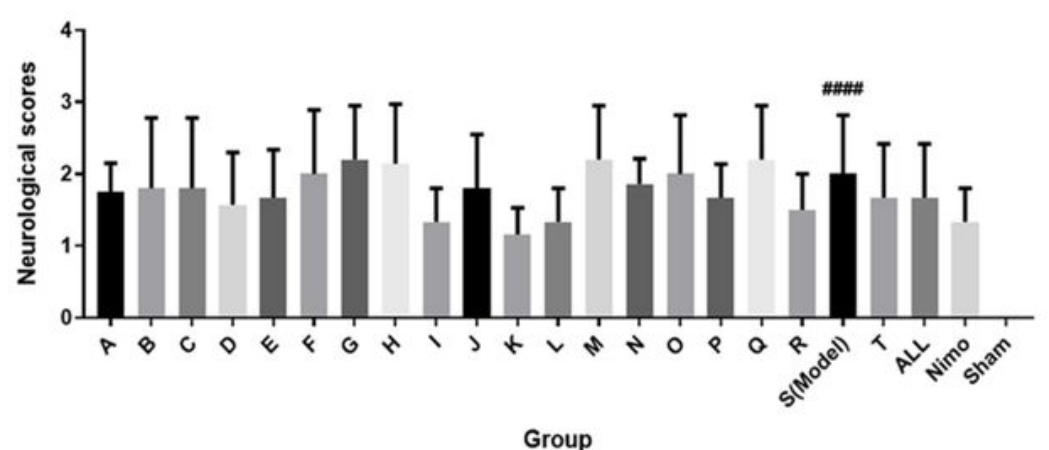

(B)
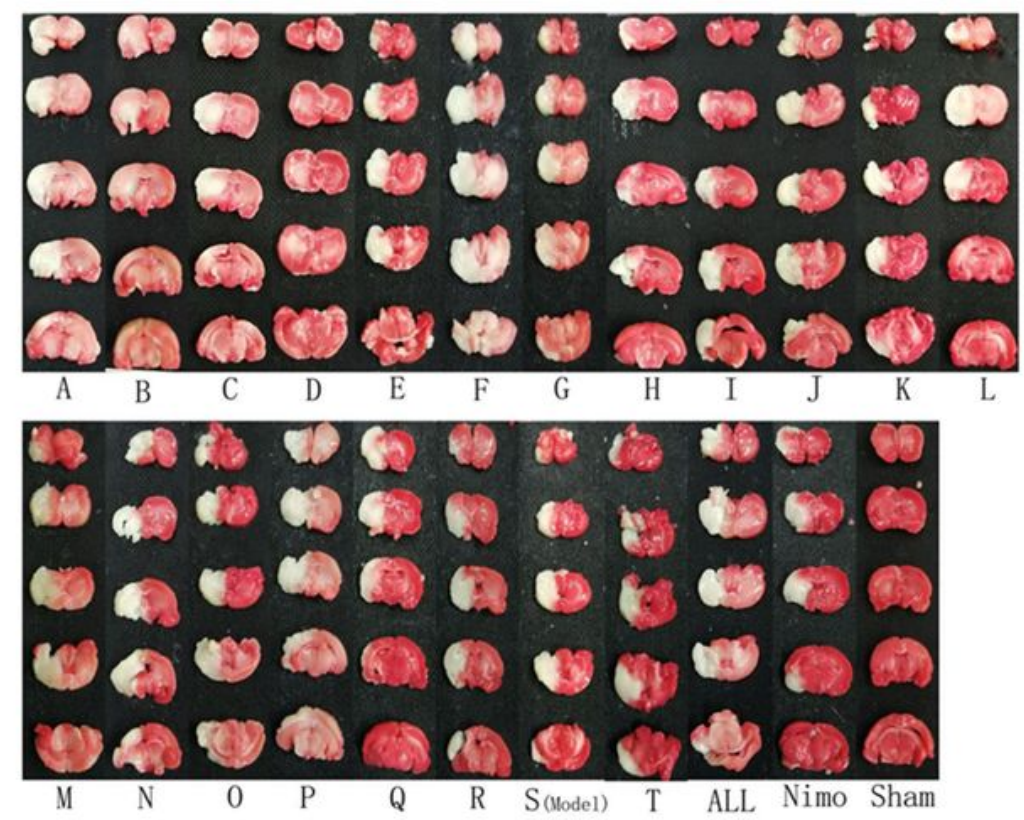

(C)

\section{Figure 2}

Results of cerebral infarction and neurological scores in each experimental group. Group A-T, the different combinations of elution fractions were selected by Plackett-Burman, $S$, model group; ALL, NMT total factor group; Nimo, Nimodipine (positive group); Sham, sham operation group. * $p<0.05$, ${ }^{\star *} p<0.01$, compared to the model group; \#\#\#\# $p<0.001$, compared to the Sham group. (A) Results of cerebral 
infarction in each experimental group, (B) Results of neurological scores in each experimental group, (C) The TTC staining of brain slices in experimental group.

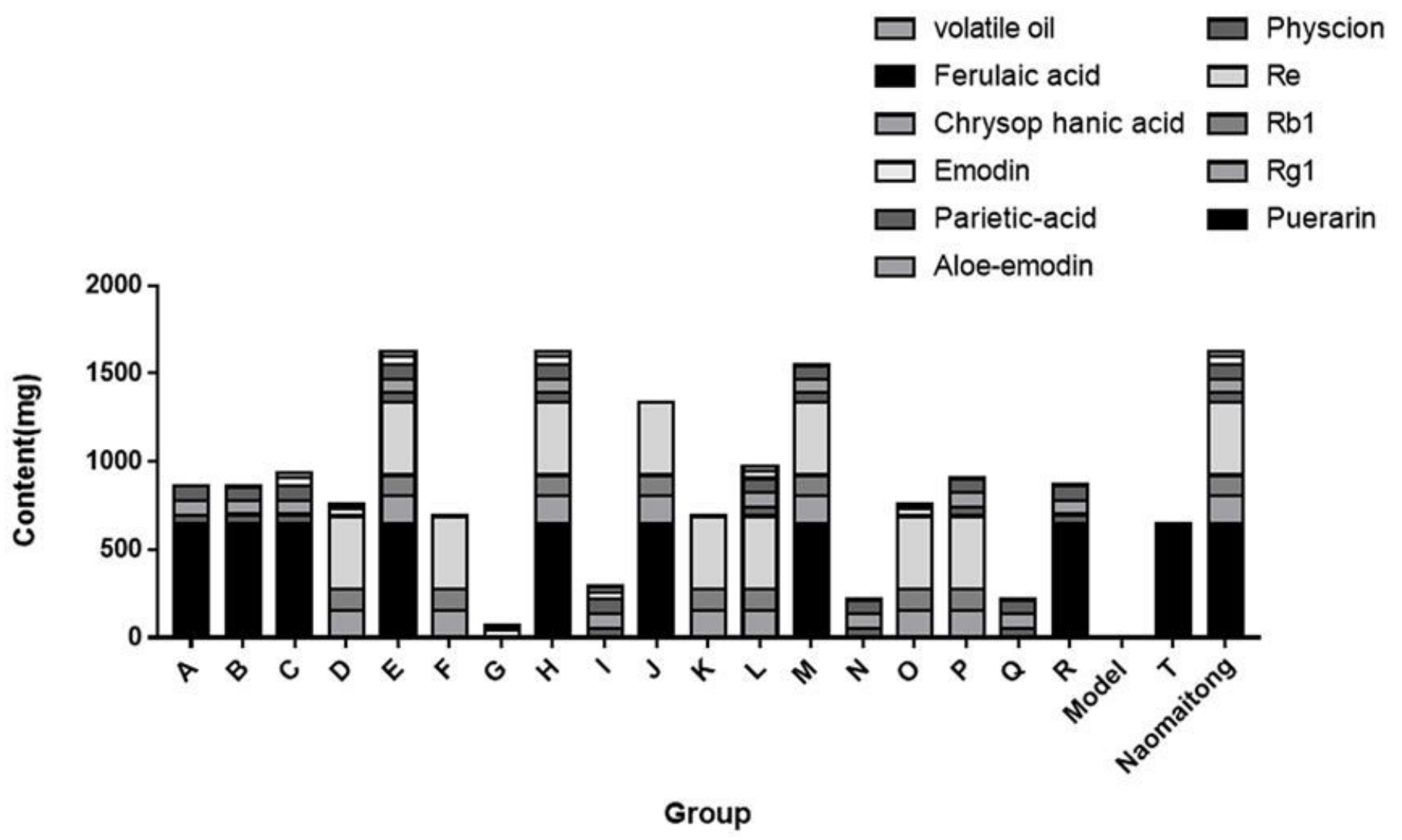

Figure 3

The content of effective components in the elution fraction Support vector machine algorithm for the screening of effective compatibility fractions of Naomaitong 


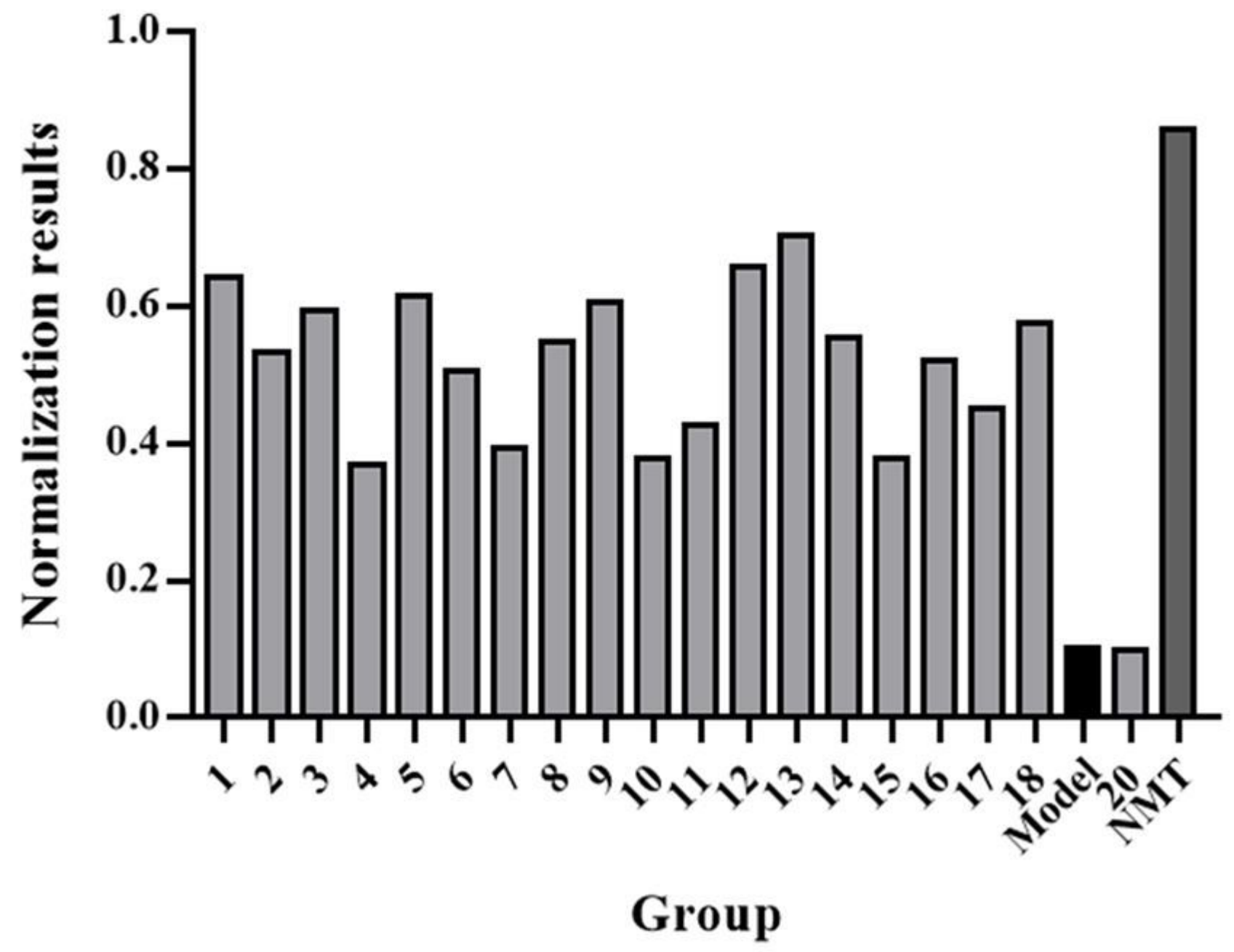

Figure 4

Normalized results of infarct size. 


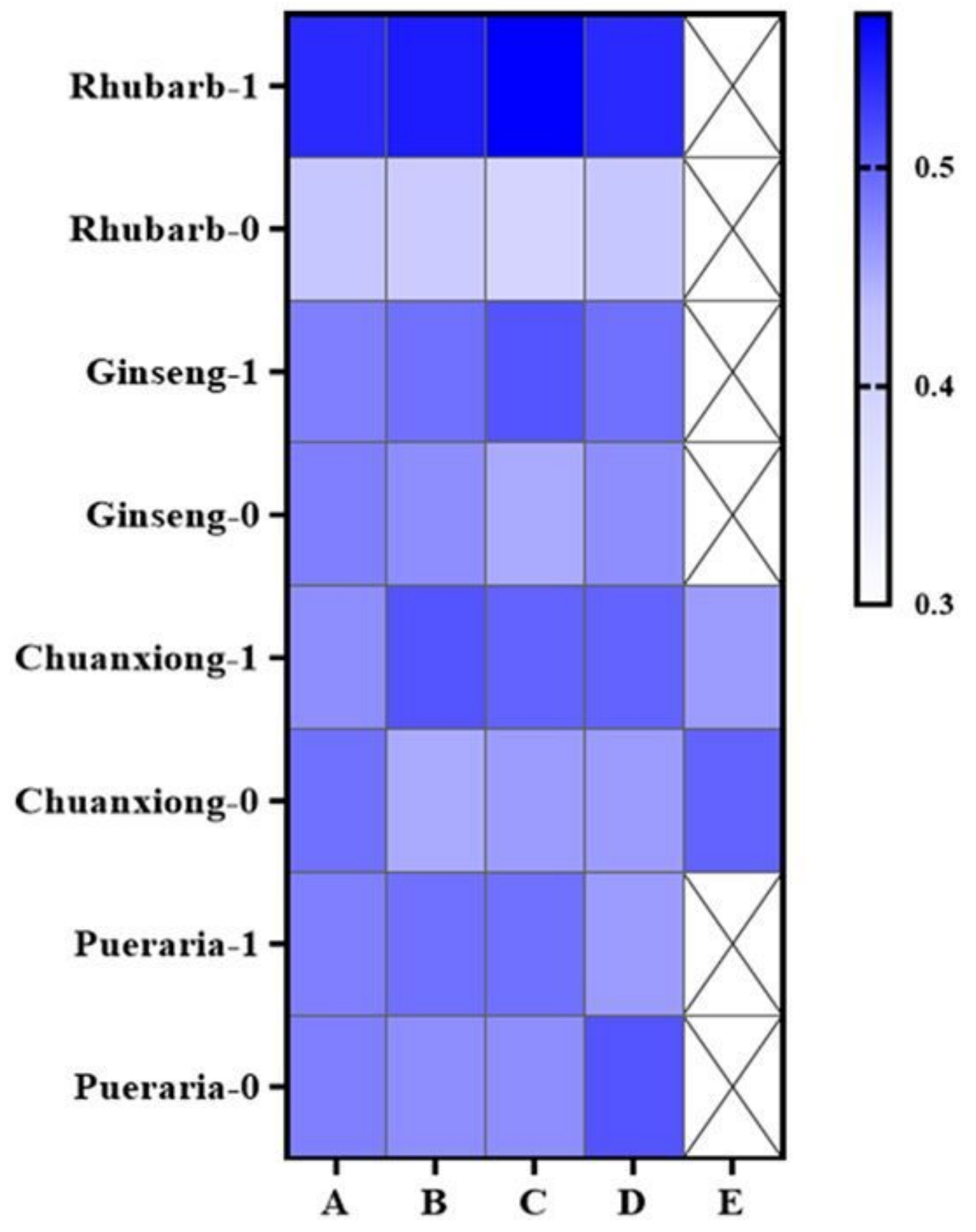

Figure 5

Heatmap of the weight coefficient at two levels for each elution component. The color indicates the weight coefficient of eluting components at two levels, and tends to be blue, indicating that the eluting component plays a more important role in compatibility. A, water elution site; B, 30\% ethanol elution site; C, $60 \%$ ethanol elution site; D, $95 \%$ ethanol elution site; E, volatile oil. 


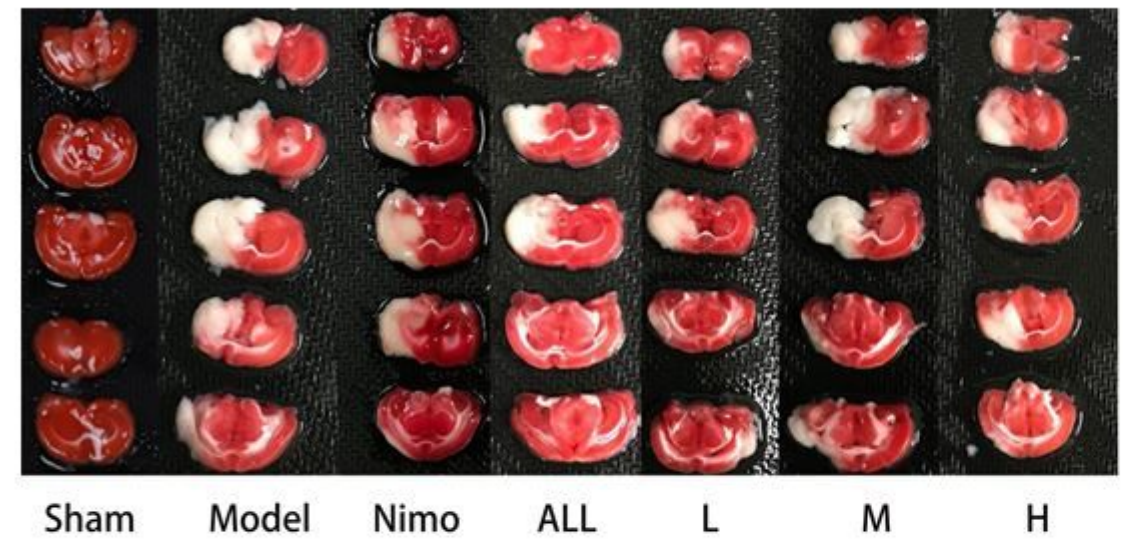

(A)

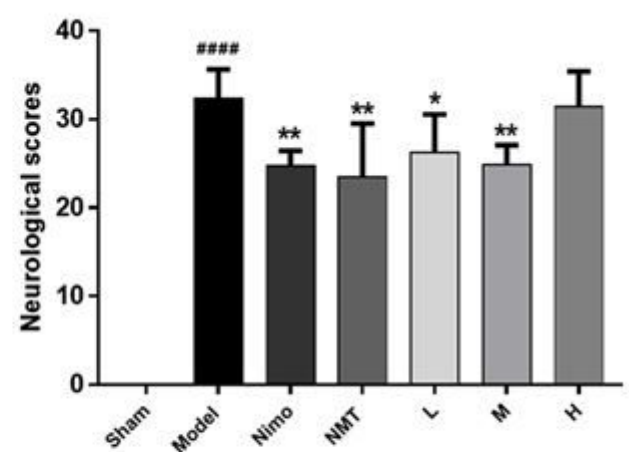

(B)

\section{Figure 6}

Verification of the brain tissue staining of each group in the experimental experiment. (A)The TTC staining of brain slices in experimental group, (B) The statistics of infarct size. ALL, NMT total factor group; $L$, low does gruop; $M$, medium does group; $H$, high does group. \#\#\# $p<0.0001$, compared to the sham operation group. ${ }^{\star} p<0.05,{ }^{\star *} p<0.01$, compared to the model group.

\section{Supplementary Files}

This is a list of supplementary files associated with this preprint. Click to download.

- SupplementaryMaterial.docx 